\title{
ALPAQUITA Array in the ALPACA Project
}

\author{
T. Asaba, ${ }^{a}$ K. Hibino, ${ }^{b}$ N. Hotta,${ }^{c}$ M. Kataoka,${ }^{a}$ Y. Katayose,${ }^{a}$ C. Kato, ${ }^{d}$ K. Kawata ${ }^{*},{ }^{e}$ \\ H. Kojima, ${ }^{f g}$ R. Mayta, ${ }^{h}$ P. Miranda,${ }^{i}$ K. Munakata, ${ }^{d}$ Y. Nakamura, ${ }^{d}$ M. Nishizawa, ${ }^{j}$ \\ S. Ogio, ${ }^{h}$ M. Ohnishi, ${ }^{e}$ A. Oshima, ${ }^{k}$ M. Raljevich, ${ }^{i}$ H. Rivera, ${ }^{i}$ T. Saito, ${ }^{l}$ T. K. Sako, ${ }^{m e}$ \\ T. Sasaki, ${ }^{a}$ S. Shibata, ${ }^{k}$ A. Shiomi,${ }^{n}$ M. Subieta,${ }^{i}$ M. Suzuki, ${ }^{a}$ N. Tajima, ${ }^{o}$ M. Takita, ${ }^{e}$ \\ Y. Tameda, ${ }^{p}$ K. Tanaka, ${ }^{q}$ R. Ticona, ${ }^{i}$ H. Tsuchiya, ${ }^{r}$ Y. Tsunesada, ${ }^{h}$ S. Udo ${ }^{b}$ and \\ M. Wakamatsu ${ }^{a}$ (The ALPACA Collaboration)
}

${ }^{a}$ Faculty of Engineering, Yokohama National University, Japan

${ }^{b}$ Faculty of Engineering, Kanagawa University, Japan

${ }^{c}$ Faculty of Education, Utsunomiya University, Japan

${ }^{d}$ Department of Physics, Shinshu University, Japan

${ }^{e}$ Institute for Cosmic Ray Research, The University of Tokyo, Japan

${ }^{f}$ Faculty of Engineering, Aichi Institute of Technology, Japan

${ }^{g}$ Chubu Innovative Astronomical Observatory, Japan

${ }^{h}$ Graduate School of Science, Osaka City University, Japan

${ }^{i}$ Instituto de Investigaciones Físicas, Universidad Mayor de San Andrés, Bolivia

${ }^{j}$ National Institute of Informatics, Japan

${ }^{k}$ College of Engineering, Chubu University, Japan

${ }^{l}$ Tokyo Metropolitan College of Industrial Technology, Japan

${ }^{m}$ Escuela de Ciencias Físicas y Nanotechnología, Yachay Tech, Ecuador

${ }^{n}$ College of Industrial Technology, Nihon University, Japan

${ }^{o}$ RIKEN, Japan

${ }^{p}$ Faculty of Engineering, Osaka Electro-Communication University, Japan

${ }^{q}$ Graduate School of Information Sciences, Hiroshima City University, Japan

${ }^{r}$ Japan Atomic Energy Agency, Japan

E-mail: kawatadicrr.u-tokyo.ac.jp

\begin{abstract}
We are now proposing a new project which consists of a large air shower array $\left(83,000 \mathrm{~m}^{2}\right)$ and a muon detector array $\left(5,400 \mathrm{~m}^{2}\right)$ located at the altitude of 4,740 $\mathrm{m}$ near La Paz in Bolivia to observe $100 \mathrm{TeV}$ gamma rays in the southern sky. The ALPAQUITA array is a prototype air shower array which will be constructed at the ALPACA site. This array consists of 45 scintillation counters of $1 \mathrm{~m}^{2}$ in area each, and its effective area is approximately $8,000 \mathrm{~m}^{2}(1 / 10$ of ALPACA air shower array). In the present paper, we report on the current status and the performance of the ALPAQUITA array.
\end{abstract}

35th International Cosmic Ray Conference - ICRC2017

10-20 July, 2017

Bexco, Busan, Korea

* Speaker. 


\section{Introduction}

The H.E.S.S. Cherenkov telescope has successfully observed diffuse component of gamma rays around the Galactic Center [四]. The spectrum of the diffuse component extends up to several tens of $\mathrm{TeV}$ with approximately power-law shape with a hard photon index $\sim 2.3$. The best-fit spectrum shape from the $\pi^{0}$ decay model suggests the existing gamma rays beyond $100 \mathrm{TeV}$. They proposed that the supermassive black hole at the Galactic Center is the most plausible source of $\mathrm{PeV}$ protons and nuclei. The H.E.S.S group also reported many of extended gamma-ray sources by the galactic plane survey [[]]. A half of sources were unidentified, and have a harder energy spectrum which is measured up to $\sim 10 \mathrm{TeV}$. On the other hand, cosmic rays are thought to be accelerated up to the knee energy region $(\sim \mathrm{PeV})$ in our galaxy. Therefore, we expect to exist cosmic gamma rays more than $100 \mathrm{TeV}$, which originate in $\pi^{0}$ decays produced by the accelerated cosmic rays interacting with matter surrounding the sources. To understand the galactic cosmic-ray origin and acceleration mechanism, a wide field-of-view gamma-ray survey in the $100 \mathrm{TeV}$ region will be a key measurement in the southern sky where the galactic sources concentrate.

We are now proposing a new project to observe 10-1000 $\mathrm{TeV}$ gamma rays with very low background noise and wide field of view in the southern hemisphere [3]. We call the ALPACA (Andes Large area PArticle detector for Cosmic ray physics and Astronomy) project. The observatory will consist of $83,000 \mathrm{~m}^{2}$ air shower array and $5,400 \mathrm{~m}^{2}$ underground water-Cherenkov-type muon detector array constructed at 4,740 m a.s.l. (middle of Mt. Chacaltaya), near La Paz in Bolivia. The gamma-ray induced air shower has much less muons compared with a cosmic-ray induced one. Therefore, the cosmic-ray background events will be significantly rejected from the gamma-ray signals by means of counting the number of muons in an air shower. With the ALPACA project, we expect to detect gamma rays between 10 and a few hundred $\mathrm{TeV}$ from the Galactic Center if cosmic rays are accelerated up to $\mathrm{PeV}$ energies at the supermassive black hole.

In advance of this full-scale ALPACA experiment, we will construct a 1/10-scale prototype scintillation detector array, so-called "ALPAQUITA". In the present paper, we report on the current status and the performance of the ALPAQUITA array.

\section{ALPAQUITA array}

The ALPAQUITA array will be constructed at an altitude of 4,740 m, near La Paz in Bolivia, which is the same as the ALPACA site. This array consists of 45 scintillation detectors of $1 \mathrm{~m}^{2}$, and the detectors are placed on a lattice with $15 \mathrm{~m}$ spacing, covering $\sim 8,000 \mathrm{~m}^{2}$ as shown in Figure $\square$ (Left).

Each counter has a plastic scintillator of $1 \mathrm{~m}^{2}\left(0.25 \mathrm{~m}^{2} \times 4\right)$ in area and $5 \mathrm{~cm}$ in thickness (made by CI Kogyo) and is equipped with a high-gain 2-inch PMT (HAMAMATSU H7195) as shown in Figure $\square$ (Right). The scintillator box is set-up in an upside-down pyramidal style, and the height of box is set to $0.7 \mathrm{~m}$ to optimize the balance between the timing accuracy and the number of observed photoelectrons. The box can be installed a low-gain PMT next to the high-gain PMT to increase dynamic range of the number of detected particles for the future plan. A $0.5 \mathrm{~cm}$-thick lead plate is put on the top of each counter to increase the array sensitivity by converting $\gamma$-rays into 


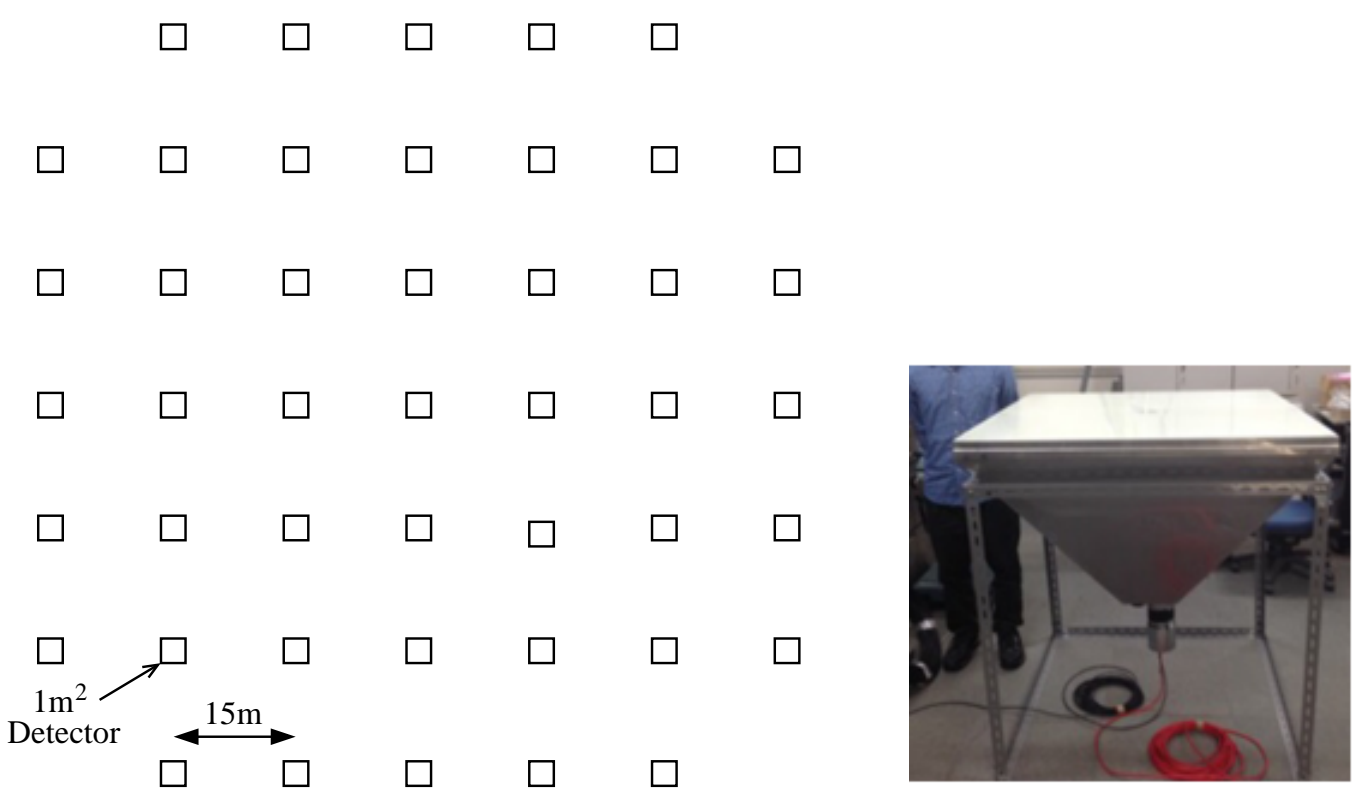

Figure 1: (Left) detector deployment of the ALPAQUITA array. Open squares show 45 scintillation detectors of $1 \mathrm{~m}^{2}$. (Right) picture of a prototype $1 \mathrm{~m}^{2}$ detector.

electron-positron pairs in the shower. The inside of all scintillator boxes is painted by white color (NIPPON PAINT ACALUX) to increase the effective photocoverage of each detector.

We have measured characteristics of a prototype detector, such as the detector gain, transit time spread, and their position dependences in the laboratory utilizing cosmic muons. The observed 1 MIP peak of the detector is clearly above dark noise, and the luminosity decreases by $\sim 10 \%$ at $50 \mathrm{~cm}$ from the center. The transit time spread of the detector is measured to be $\sim 0.7 \mathrm{~ns}(\sim 1.1 \mathrm{~ns})$ at the center of detector (at $50 \mathrm{~cm}$ from the center). Based on these detector characteristics, we are now developing the detailed MC simulation.

The electronics hut will be constructed around center of the array. The whole detector is covered with a white sheet to reduce the temperature variation. All the signal cables of detectors have the same length of $100 \mathrm{~m}$ and use low-loss and high-frequency coaxial cable (FUJIKURA 2.5D-HQ.SUPER).

\section{Performances and Sciences}

The primary purpose of the ALPACA experiment is the gamma-ray astronomy at the energy range between $10 \mathrm{TeV}$ and $1000 \mathrm{TeV}$ in the southern sky. In addition, we will also observe the charged cosmic rays with a threshold of a few TeV. The ALPAQUITA array will be operated as an engineering air shower array without the muon detectors. Using this array, we will evaluate the performance, and develop the MC simulation, and expect some traditional cosmic-ray studies.

Recently, the IceCube and IceTop experiments located at the South Pole measured the detailed cosmic-ray anisotropy at the energies $20-2000 \mathrm{TeV}$ in the southern sky [ $[$, [5]. They found changes of anisotropy features depending on the energy. Currently, there is no anisotropy measurement at 
the $\mathrm{TeV}$ region in the southern hemisphere. The ALPAQUITA array can first measure the cosmicray anisotropy above a few $\mathrm{TeV}$ in the southern sky. The trigger rate of cosmic ray is estimated to be roughly $150 \mathrm{~Hz}$. We expect to detect the large-scale sidereal anisotropy with an amplitude $\sim 0.1 \%$ at the significance $\sim 10 \sigma /$ year.

The Tibet air shower experiment have continuously observed the Sun's shadow since 1990 [回] in the northern hemisphere. They found a clear solar-cycle variation of the Sun's shadow is seen in the $10 \mathrm{TeV}$ during a full solar cycle from 1996 to 2009 [ [U]. Since the site of the ALPAQUITA is located near the equator, the culmination altitude of the Sun is relatively high. Therefore, the Sun's shadow observation is possible through 1 year in our FoV with the zenith angle less than $50^{\circ}$. Thus, the exposure of the ALPAQUITA along the Sun's orbit will be twice larger than that of the identical array placed in Tibet.

\section{Summary}

The ALPAQUITA array is a prototype air shower array which will be constructed at the ALPACA site. This array will consist of 45 scintillation counters of $1 \mathrm{~m}^{2}$ in area each, and its effective area is approximately $8,000 \mathrm{~m}^{2}$ (1/10 of ALPACA air shower array). The preparations of site infrastructures, detectors and the DAQ electronics are on going for this new project. In the current schedule, the ALPAQUITA array will start the data taking, at the end of 2017 or the beginning of 2018, to observe multi TeV cosmic rays in the southern sky.

\section{Acknowledgments}

The authors would like to thank the staff of the Instituto de Investigaciones Físicas, Universidad Mayor de San Andrés, La Paz, Bolivia, for their support to our experiment at Mount Chacaltaya. This work is supported by the joint research program of the Institute for Cosmic Ray Research (ICRR), The University of Tokyo. KK is supported by the Toray Science Foundation.

\section{References}

[1] A. Abramowski, et al., Acceleration of petaelectronvolt protons in the Galactic Centre, Nature, 531, 476 (2016).

[2] F. Aharonian, et al., The H.E.S.S. survey of the Inner Galaxy in very high-energy gamma-rays, ApJ, 636, 777 (2006).

[3] T. Asaba, et al., The overview of the ALPACA Experiment, these proceedings (2017).

[4] R. Abbasi, et al., Observation of an Anisotropy in the Galactic Cosmic Ray arrival direction at 400 TeV with IceCube, ApJ, 746, 33 (2012).

[5] M.G. Aartsen, et al., Observation of Cosmic Ray Anisotropy with the IceTop Air Shower Array, ApJ, 765, 55 (2013).

[6] M. Amenomori, et al., Direct Evidence of the Interplanetary Magnetic Field Effect on the Cosmic-Ray Shadow by the Sun, ApJ, 415, L147 (1993).

[7] M. Amenomori, et al., Probe of the Solar Magnetic Field Using the "Cosmic-Ray Shadow" of the Sun, PRL, 111, 011101 (2013). 